\title{
Crystal structure of catena-octachlorotrisiloxane, $\mathrm{Si}_{3} \mathrm{O}_{2} \mathrm{Cl}_{8}$
}

\author{
M. Binnewies ${ }^{*, I}$ and H. Borrmann ${ }^{I I}$ \\ I Universität Hannover, Institut für Anorganische Chemie, Callinstr. 9, D-30167 Hannover, Germany \\ II Max-Planck-Institut für Chemische Physik fester Stoffe, Nöthnitzer Str. 40, D-01187 Dresden, Germany
}

Received June 4, 2002, accepted and available on-line August 6, 2002; CSD-No. 409627

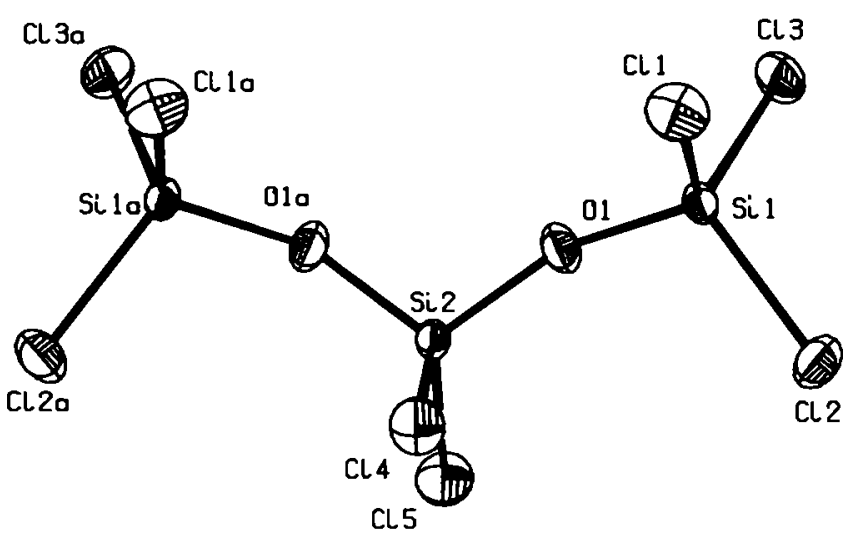

Abstract

$\mathrm{Cl}_{8} \mathrm{O}_{2} \mathrm{Si}_{3}$, orthorhombic, Pnma (No. 62), $a=10.669(1) \AA$, $b=20.317(3) \AA, c=6.183(1) \AA, V=1340.4 \AA^{3}, Z=4$, $R_{\mathrm{gt}}(F)=0.023, w R_{\mathrm{obs}}\left(F^{2}\right)=0.055, T=90 \mathrm{~K}$.

\section{Source of material}

The preparation method for chlorosiloxanes $\mathrm{Si}_{\mathrm{x}} \mathrm{O}_{\mathrm{y}} \mathrm{Cl}_{\mathrm{z}}$ is described in the literature [1]. $\mathrm{Si}_{3} \mathrm{O}_{2} \mathrm{Cl}_{8}$ can be isolated and purified by fractional destillation. The compound is highly moisture sensitive.

\section{Discussion}

High temperature reaction between siliconchloride $\left(\mathrm{SiCl}_{4}\right)$ and oxygen leads to $\mathrm{SiO}_{2}$. At about $1300 \mathrm{~K}$ chlorosiloxanes like $\mathrm{Si}_{3} \mathrm{O}_{2} \mathrm{Cl}_{8}$ are formed as intermediates during this reaction.

The molecular structure of these compounds are of particular interest regarding the pathway from molecular $\mathrm{SiCl}_{4}$ to solid $\mathrm{SiO}_{2}$. $\mathrm{Si}_{3} \mathrm{O}_{2} \mathrm{Cl}_{8}$ shows the expected chain structure with bridging oxygen atoms.
Table 1. Data collection and handling.
Crystal:

Wavelength:

$\mu$ :

Diffractometer, scan mode:

$2 \theta_{\max }$ :

$N(h k l)_{\text {measured, }} N(h k d)_{\text {unique: }}$

Criterion for $I_{\mathrm{obs}}, N(h k l)_{\mathrm{gt}}$ :

$N($ param) refinod:

Programs: colourless needle, size $0.2 \times 0.2 \times 1.0 \mathrm{~mm}$

$\mathrm{Ag} K_{\alpha}$ radiation (0.56086 $\AA$ )

$9.65 \mathrm{~cm}^{-1}$

Syntex $P 21, \omega$

$52.14^{\circ}$

5622,2769

$I_{\mathrm{obs}}>2 \sigma\left(I_{\mathrm{obs}}\right), 2266$

64

SHELXS-86 [2], SHELXI-93 [3],

PLATON [4]

\section{References}

1. Quellhorst, H.; Wilkening, A.; Binnewies, M.: Trennung und massenspektrometrische Charakterisienung von Perchlorsiloxanen. Z. Anorg. Allg. Chem. 623 (1997) 1871-1874.

2. Sheldrick, G. M.: SHEL XS-86. A program for crystal structure determination, University of Göttingen, Germany 1990.

3. Sheldrick, G. M.: SHEL XI-93. A program for refining crystal structures, University of Göttingen, Germany 1993.

4. Spek, A. L.: PLATON, an integrated tool for the analysis of the results of a single crystal structure determination. Acta Crystallogr. Suppl. A46 (1990) C-34.

Table 2. Atomic coordinates and displacement parameters (in $\AA^{2}$ ).

\begin{tabular}{|c|c|c|c|c|c|c|c|c|c|c|}
\hline Atom & Site & $x$ & $y$ & $z$ & $U_{11}$ & $U_{22}$ & $U_{33}$ & $U_{12}$ & $U_{13}$ & $U_{23}$ \\
\hline $\mathrm{Si}(1)$ & $8 d$ & $0.91472(3)$ & $0.38581(1)$ & $0.11360(4)$ & $0.0195(1)$ & $0.0133(1)$ & $0.0187(1)$ & $-0.00166(9)$ & $-0.00075(9)$ & $-0.00163(8)$ \\
\hline $\mathrm{Si}(2)$ & $4 c$ & $0.79252(3)$ & $1 / 4$ & $0.02150(6)$ & $0.0160(2)$ & $0.0120(2)$ & $0.0186(2)$ & 0 & $0.0000(1)$ & 0 \\
\hline $\mathrm{Cl}(1)$ & $8 d$ & $1.07992(3)$ & $0.38149(2)$ & $-0.04216(5)$ & $0.0248(1)$ & $0.0437(2)$ & $0.0335(1)$ & $-0.0037(1)$ & $0.0086(1)$ & $-0.0005(1)$ \\
\hline $\mathrm{Cl}(2)$ & $8 d$ & $0.79685(3)$ & $0.44556(2)$ & $-0.04713(5)$ & $0.0414(2)$ & $0.0256(1)$ & $0.0314(1)$ & $0.0109(1)$ & $-0.0096(1)$ & $0.0010(1)$ \\
\hline $\mathrm{Cl}(3)$ & $8 d$ & $0.94030(3)$ & $0.41963(2)$ & $0.41488(4)$ & $0.0367(1)$ & $0.0267(1)$ & $0.0221(1)$ & $-0.0011(1)$ & $-0.0049(1)$ & $-0.00781(9)$ \\
\hline $\mathrm{Cl}(4)$ & $4 c$ & $0.81927(4)$ & $1 / 4$ & $-0.30012(6)$ & $0.0388(2)$ & $0.0321(2)$ & $0.0189(2)$ & 0 & $0.0026(1)$ & 0 \\
\hline $\mathrm{Cl}(5)$ & $4 c$ & $0.60814(4)$ & $1 / 4$ & $0.08485(8)$ & $0.0170(1)$ & $0.0348(2)$ & $0.0437(2)$ & 0 & $0.0054(1)$ & 0 \\
\hline$O(1)$ & $8 d$ & $0.85557(8)$ & $0.31359(4)$ & $0.1295(1)$ & $0.0306(4)$ & $0.0160(3)$ & $0.0272(3)$ & $-0.0063(3)$ & $-0.0028(3)$ & $-0.0011(3)$ \\
\hline
\end{tabular}

\footnotetext{
* Correspondence author (e-mail: binn@mbox.aca.uni-hannover.de)
} 